\title{
Robust Control of Maintenance-Phase Anesthesia
}

\author{
JOÃO M. LEMOS, DANIELA V. CAIADO, BERTINHO A. COSTA, LUIS A. PAZ, TERESA F. MENDONÇA, \\ RUI RABIÇO, SIMÃO ESTEVES, and MANUEL SEABRA
}

n $\mathrm{n}$ biomedical systems, feedback control can be applied whenever adequate sensors, actuators, and sufficiently accurate mathematical models are available. The key issue is the capacity of the control algorithm to tackle the large levels of uncertainty, both structured and unstructured, associated with patient dynamics. In the particular case of intravenous anesthesia considered here, manipulated variables are drug infusion rates, administered by syringe pumps, and the measured signal outputs are the levels of hypnosis or depth of anesthesia (DoA) and of neuromuscular blockade (NMB). Figure 1 provides an example of a loop closed for the control of NMB.

In addition to these indices that are to be kept close to desired target reference values, the medical anesthetist has to monitor the general physiological state of the patient [1]. This task is performed with equipment of the sort shown in Figure 2 that provides information about patient heart rate, arterial blood pressure, and oxygen transport.

No attempt is made here to make a review of the rich literature on control of anesthesia, and only a few landmarks that help explain the motivation to develop the case studies presented in this article are cited. Since the late 1980s, several control techniques have been described for drug administration in anesthesia with applications to NMB, such as a simple on-off controller based on a relay and a syringe pump [2], or proportional-integral-derivative (PID) controllers [3], [4]. More complex PID controllers with self-tuning methods that adjust the controller gains to the patient's dynamics have also been reported for NMB [5]-[7]. To maintain the hypnosis level, PID controllers are reported as well [8], along with model-based control techniques [9]-[12].

The high variability in anesthesia dynamics, both interand intrapatient, may cause performance degradation and even instability of the control loop. To tackle this difficulty, there are basically two broad classes of approaches that can be followed, namely adaptive control and robust control.

Adaptive control techniques, based on the pharmacokinetics/phamacodynamic (PK/PD) model [13]-[16] perform better than PID controllers to maintain the desired NMB

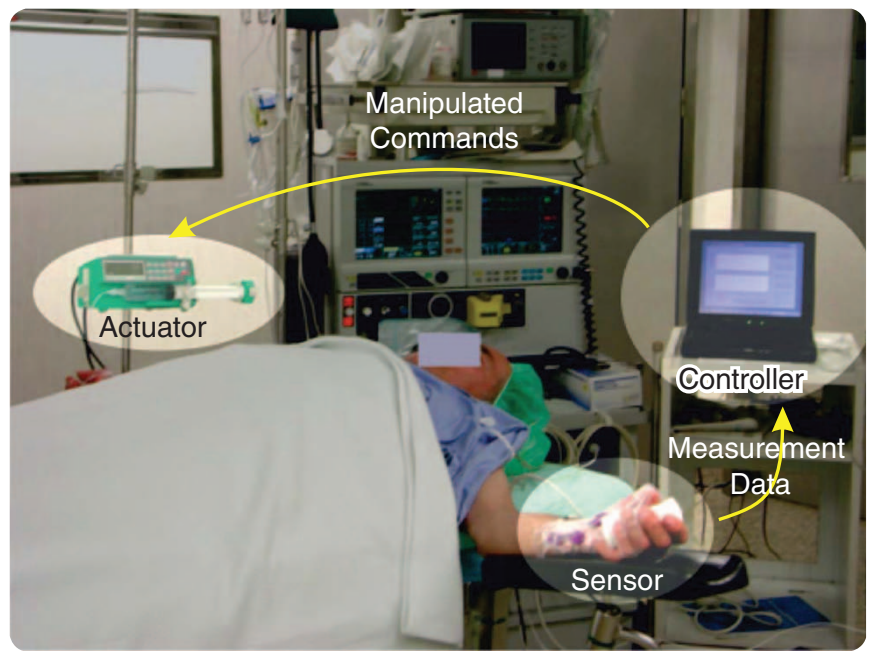

FIGURE 1 Closing the neuromuscular blockade (NMB) control loop in a patient subject to general anesthesia. Data on the NMB level are transmitted to the controller in real time, with a sampling rate of $20 \mathrm{~s}$. The control algorithm is embedded in a software package that runs in the computer. From the data received from the sensor, the control algorithm computes the necessary drug dosage to administer to the patient to keep the NMB level at a desired setpoint and communicates the dosage to a syringe pump that functions as the actuator. The interface of the computer with the sensor and the syringe pump is made through USB ports that emulate RS232 interfaces.

level. More elaborate model-based adaptive feedback control algorithms have been reported, such as multimodel adaptive controllers with supervisory mechanisms [17], [18]. Adaptive control strategies for hypnosis have also been described [19][21]. Methods that explore the nonnegative and compartmental features of the physiological models used in anesthesia to develop both fixed parameter and adaptive controllers are highlighted in [22]. However, since, in addition to the output feedback loop, adaptive controllers have a nonlinear feedback loop associated with the adaptation mechanism, the resulting closed loop becomes nonlinear and can exhibit undesirable behaviors that are often difficult to anticipate. This is due to parameters drifting over manifolds that are difficult, or even impossible, to characterize. In particular, for several adaptive controllers that result from the certainty equivalence principle [23], a complete stability theory is not available. The above issues motivate, in relation to the control of anesthesia, 
the consideration of linear constant-parameter controllers that are able to withstand the high levels of uncertainty that are inherent to a biomedical process. This type of design is in the scope of robust control, of which [24] and [25] are early attempts. Although, in the case of single-input, single-output plants, robust pole placement controllers can be designed using a trial-and-error method [26], it is advantageous to use a systematic procedure such as the $H_{\infty}$ method [27]. An alternative approach would be to design a fixed-parameter controller by applying the techniques described in [22]. In this article, a constant linear controller designed by the $H_{\infty}$ method is employed to compute the control action, thereby avoiding the drawbacks of adaptive control caused by gain drifting.

\section{ANESTHESIA AS A CONTROL PROBLEM}

In medical practice, the application of general anesthesia plays an important role in the patient's well-being, allowing surgical interventions without discomfort or pain and providing surgeons with the conditions to perform with increased accuracy and safety.

General anesthesia is a drug-induced, reversible state characterized by unconsciousness, immobility, and inability to feel pain [28]. This patient state is achieved through the administration of a combination of different drugs that act to provide adequate hypnosis (unconsciousness and amnesia to avoid traumatic recalls), paralysis or muscle relaxation (to attain immobility, absence of reflexes, and good operating conditions), and analgesia (pain relief).

The automation of general anesthesia has the purpose of achieving a sufficient state of hypnosis, paralysis, and analgesia while minimizing drug quantities and side effects. Drug overdosing or underdosing may lead to undesirable side effects. For instance, underdosage of neuromuscular relaxant drugs often interferes with the surgical precision, while overdosage delays patient recovery. Underdosing hypnotic agents may result in patient awareness and cause psychological trauma, whereas overdosing may be harmful with respect to possible perioperative morbidity. An important consideration relating the induction and maintenance of anesthesia is therefore patient safety to which automation contributes. Only the maintenance phase of anesthesia is addressed in this article.

The characterization of anesthesia in the maintenance phase as a control problem is described below.

For NMB, the manipulated variable is the flow of a myorelaxant drug, like rocuronium. This drug perfusion is made with syringe pumps that are connected to a computer that runs the control algorithm via USB interfaces, as shown in Figure 3. The process output is the NMB index measured with a mechanical sensor (see "Neuromuscular Blockade Monitoring and NMB Indices"). Both the manipulated variable and the process output are related by a Wiener-type model, as described in "Neuromuscular Blockade and Hypnotic Models." Although the NMB index is subject to several disturbances, these influences are considered not to be directly measurable. For instance, the electrical bistoury affects the

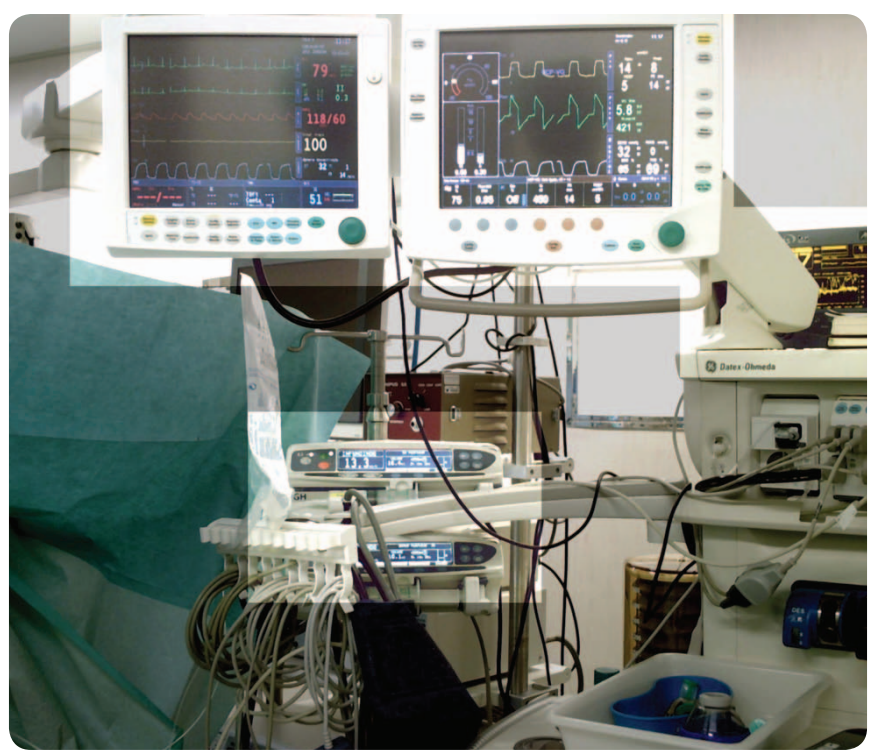

FIGURE $2 \mathrm{~A}$ view of the monitors in the operating room. Commercially available monitors display important information concerning the state of the patient who is undergoing surgery, both in numerical and graphical form. A large amount of diverse information can be monitored and the type and form of the information displayed results from a compromise between characterizing the patient's clinical state and the capacity of the anesthetist to correctly read and interpret the data, particularly during critical situations.

NMB index, as do some drugs that are administered to the patient, but the signals that represent these influences are not available and the models associated with them are unknown; hence they are considered to be unmeasured disturbances.

For DoA, the manipulated variable is the flow of a hypnotic drug like propofol, also administered to the patient intravenously using a syringe pump. In the case

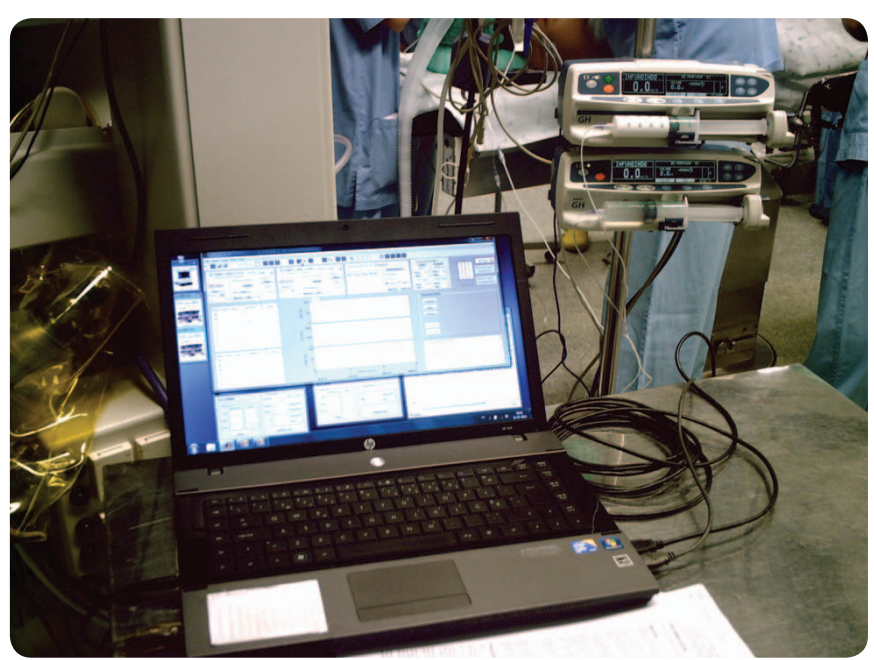

FIGURE 3 The computer used to run the control algorithms and the syringe pumps used in the tests (upper right). One syringe pump was used to administer the neuromuscular blockade-inducing drug, and the other syringe pump was used to administer the hypnotic drug. The analgesic drug is administered by the anesthetist using a manual syringe. At any time, for safety purposes, the anesthetist can turn off the automatic control action and command any syringe pump directly in manual mode. 


\section{Neuromuscular Blockade Monitoring and NMB Indices}

euromuscular blockade (NMB) monitoring of a patient subject to general anesthesia [S1], [S2], in a hypnotic and analgesic state, is used to evaluate the level of muscle relaxation induced by drugs. NMB monitoring has three main purposes. The first is to ensure appropriate relaxation of vocal cords and neck muscles to allow safe tracheal intubation and mechanical ventilation. The second is to assess the muscle tonus during surgery, allowing for adjustment to particular surgical requirements of immobility and muscle relaxation. The third, at the end of the anesthesia, is to assess the level of residual NMB and muscle strength to decide the timing of tracheal extubation and assumption of spontaneous autonomous ventilation.

The principle of NMB monitoring consists of the electrical stimulation of a motor nerve and the evaluation of the induced muscle response level [S1], [S2]. Usually the ulnar nerve at the wrist is used, where electrodes are applied over the cleaned skin above the nerve. A calibration process is performed initially to find the electrical current intensity that corresponds to the supra maximal stimulation, which typically ranges between 20 and $70 \mathrm{~mA}$. The electrical stimulation can be generated by just one rectangular pulse with a duration of $0.2 \mathrm{~ms}$ (single twitch) and a frequency of $0.1-1 \mathrm{~Hz}$ [S3]. Other possibilities are the train-of-four (TOF), the double-burst stimulation, and the tetanic stimulation [S4], [S5]. As shown in Figure S1, the TOF is characterized by a sequence of four pulses (twitches) at $0.5 \mathrm{~s}$ intervals. The train of pulses is repeated with a TOF period that can be selected and is typically $20 \mathrm{~s}$, a value that ensures the recovery of the muscle to its unstimulated state. The TOF method is adequate for the assessment of the
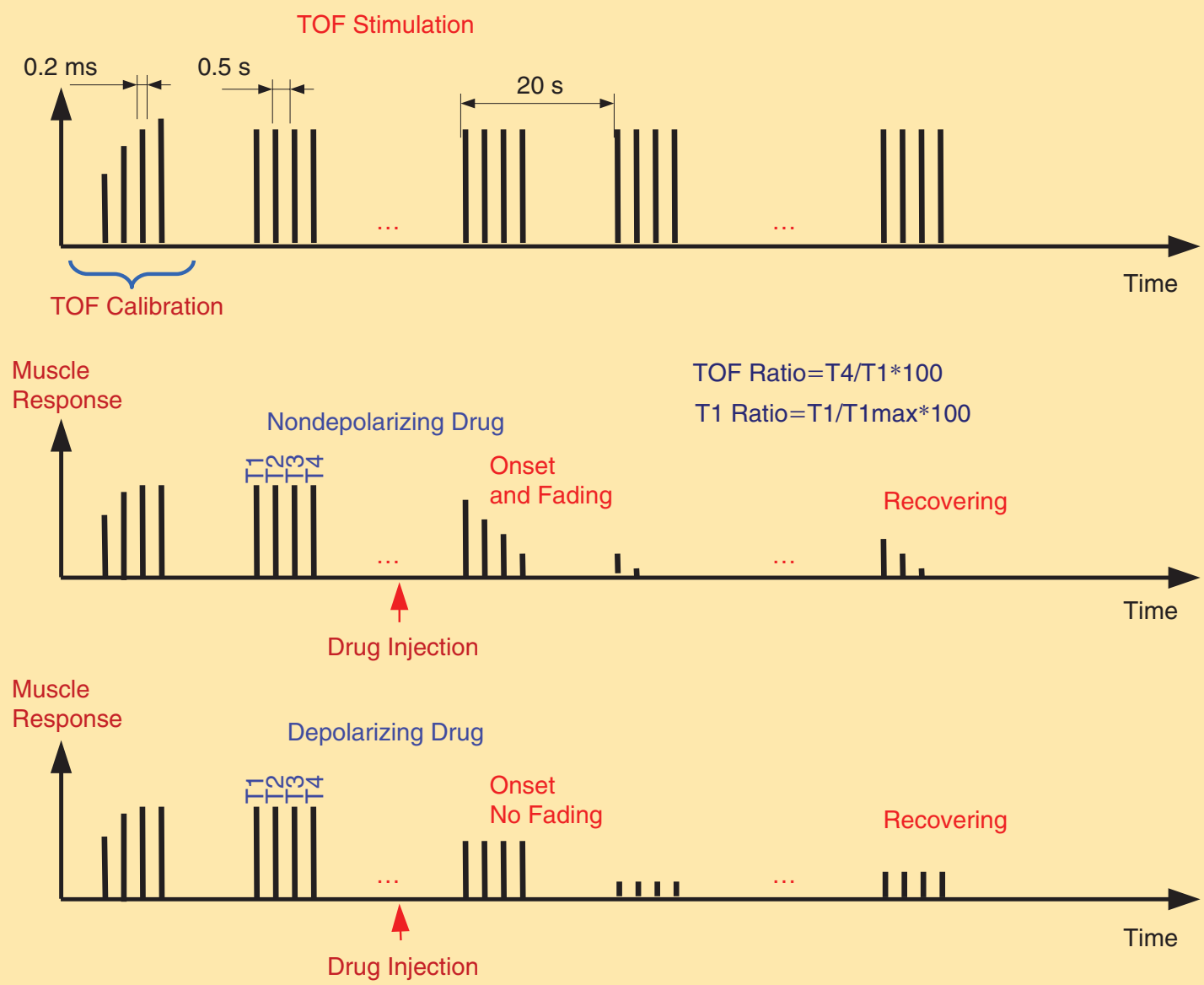

FIGURE S1 An illustration of neuromuscular blockade (NMB) indices based on train-of-four (TOF) stimulation and the muscle response with nondepolarizing and depolarizing NMB agents.

considered here, the process output is the bispectral (BIS) index (see "Monitoring the Depth of Anesthesia (DoA)"). The analgesic drug (in this case, remifentanil) interferes with the effect caused by the hypnotic drug. Although the analgesic drug flow could therefore be used to cre- ate a feedforward effect, this approach is not pursued here. The model that relates the manipulated variable, the input disturbance associated to the analgesic drug, and the process output is described in "Neuromuscular Blockade and Hypnotic Models." 
onset of action of the NMB drug, for nondeep NMB monitoring when there are TOF responses, and for neuromuscular recovery. But for deep neuromuscular blockade, when TOF responses are not present, tetanic stimulation must be used.

Several methods can be used to measure the intensity of the muscle response to electrical pulse stimulation [S6]. In the mecanomyography method, the force developed during the isometric contraction of the adductor policis muscle is recorded. In the electromyography method, electrodes are used to record the muscle electrical activity. In acceleromyography, the acceleration of the thumb is recorded. In phonomyography, low-frequency acoustic signals emitted by a muscle in contraction are recorded and analyzed.

The muscle response to TOF stimulation pulses, in patients who do not have neuromuscular diseases, depends on the type of NMB drug used and on the time-varying drug concentration in the blood. With the onset of NMB with nondepolarizing NMB agents, the responses to TOF pulses have a fade characteristic, their amplitude decreases with drug concentration, and the response to the fourth pulse is lower than the response to the third pulse, which is lower to the response of the second pulse, and so on. This phenomenon is explored to define indices, either by taking the ratio between the fourth response to the first response, the TOF ratio $=100 T_{4} / T_{1}$, or by computing the ratio between the first response and the response obtained with the supra maximal stimulation during the calibration, given by the $T_{1}$ ratio $=100 T_{1} / T_{1 \max }$. It has been demonstrated [S7] that for a $90 \%$ suppression of $T_{1}$, compared with the supra maximal stimulation response, only one response will be observed. During surgery, and according to clinical requirements, the $T_{1}$ ratio is kept equal or lower than $10 \%$. As an additional index, the TOF count, consisting in the counting of responses to a train of four pulses, is also used by anesthetists.

In the case of depolarizing NMB drugs, the depolarizing block has two phases. In phase I, the fading characteristic is not present, but for some patients the fade occurs (phase II) with increasing depolarization. With depolarizing NMB agents, the TOF ratio is meaningless, but the $T_{1}$ ratio index can be computed.

During surgery, the level of the $T_{1}$ ratio is adjusted (through drug administration) to suppress muscle movements with the minimal amount of drug, but near the end of the surgery, it must be adjusted to allow a rapid recovering of the muscle activity.

In this work, the Datex-Ohmeda NMB monitoring system shown in Figure S2 was configured to operate in the TOF mode with the TOF period of $20 \mathrm{~s}$. This value is selected as a balance between the selection of the sampling time for the NMB control system and the time needed for rebuilding of acethylcholine in the nerve ending. The muscle responses are measured with a Datex

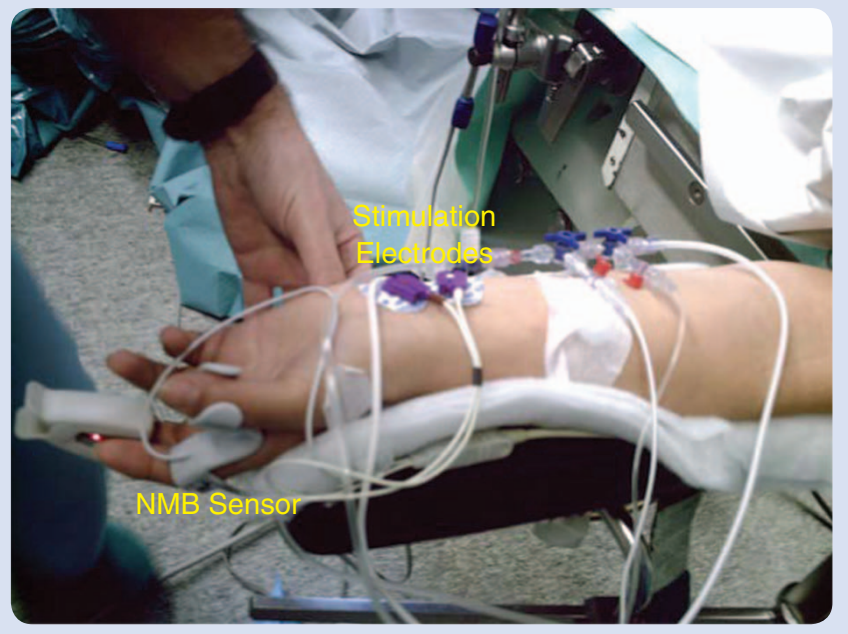

FIGURE S2 The neuromuscular blockade (NMB) sensor. This photograph is of the Datex-Ohmeda NMB monitoring with stimulation electrodes applied over the ulnar nerve and the NMB sensor applied to measure thumb movements. The force sensor that measures the contraction of the thumb induced by the electrical stimulation.

NMT mechanosensor, in which the bending of the thumb produces a voltage signal from a piezoelectric. The $T_{1}$ ratio is used as the NMB index and the TOF count is also available to the anesthetist.

In practice, any NMB index has noise and artifacts caused by procedures performed by the anesthetist, nurse practitioners, and the surgical team. To use the NMB signal in a closedloop control system, a median filter combined with low-pass filters can be used to attenuate the artifact effects in the NMB signal. Median filters are used to cancel outliers generated by non-Gaussian noise with a heavy tail distribution.

\section{REFERENCES}

[S1] G. E. Morgan, M. S. Mikhail, and M. J. Murray, Clinical Anesthesiology, 4th ed. New York: McGraw-Hill, 2006.

[S2] T. Fuchs-Buder, Neuromuscular Monitoring in Clinical Practice and Research. Berlin Heidelberg, Germany: Springer-Verlag, 2010.

[S3] M. D. Fiore, J. L. Atlee, J. G. Webster, and W. J. Tompkins, "A microcomputer-based neuromuscular blockade monitor," IEEE Trans. Biomed. Eng., vol. 28, pp. 775-783, Nov. 1981.

[S4] T. M. Hemmerling and N. Le, "Brief review: Neuromuscular monitoring: An update for the clinician," Can. J. Anaesth., vol. 54, no. 1, pp. 58-72, 2007.

[S5] T. Fuchs-Buder, J. U. Schreiber, and C. Meistleman, "Monitoring neuromuscular block: An update," Anaesthesia, vol. 64, pp. 82-89, Mar. 2009. [S6] G. Trager, S. Michaud, S. Deschamps, and T. M. Hemmerling, "Comparison of phonomyography, kinemyography and mechanomyography for neuromuscular monitoring," Can. J. Anesth., vol. 53, pp. 130135, Dec. 2006

[S7] C. D. McGrath and J. M. Hunter, "Monitoring of neuromuscular block," Continuing Edu. Anaesth., Critical Care Pain, vol. 6, no. 1, pp. 7-12, Feb. 2006.
Since reliable indices to measure analgesia do not exist, this component of anesthesia is not automated. Furthermore, only intravenous anesthesia is considered in this article, leaving aside the important area of inhalation anesthesia.

\section{MODELS FOR CONTROL OF ANESTHESIA}

Despite the complexity of the mechanisms for drug absorption, distribution through the patient body, metabolism, and elimination, it is possible from a control engineering point of view to use phenomenological-type models that relate the 


\section{Monitoring the Depth of Anesthesia (DoA)}

- he traditional medical assessment of DoA is based on a qualitative assessment of clinical signs, such as sweating, body movement, and changes in the blood pressure or heart rate. These qualitative measures allow the anesthetist to verify the depth of anesthesia but are not adequate for closed-loop control. Thus, other sources of information have been explored to assess DoA, such as the recording of electrical activity along the scalp (electroencephalography), either by processing the autonomous electroencephalogram (EEG) signal or by using evoked potentials and seeking the corresponding pattern in the EEG signal, as in the auditory evoked potential.

When a person is awake and alert with open eyes, the EEG has beta waves characterized by a frequency between 13 and $30 \mathrm{~Hz}$. In a relaxed state with the eyes closed, the EEG is characterized by waves with frequency components between 8 and $13 \mathrm{~Hz}$. Above $30 \mathrm{~Hz}$, the EEG has components from the electrical activity produced by skeletal muscles as well as from other sources, such as from the main power system at $50-60 \mathrm{~Hz}$. Thus, the EEG must be filtered to remove artifacts. Research has shown that humans are able to describe dreams that they have during rapid eye movement (REM) sleep, which corresponds to cognitive activity and is associated with desynchronous EEG of small amplitude and high frequency content. Absence of cognitive functions is associated with synchronous EEG that has a high amplitude and low frequency content.

Desynchronous EEG is observed during hypnotic states induced in patients with anesthetic drugs [28], [S8]. An increase of beta waves occurs during the initial stages of sedation, amnesia, and anxiolsys, with drugs such as benzodiazepines and barbiturates. Increasing anesthetic drug doses causes synchronous EEG during anesthesia. If drug concentration in the brain becomes too high, then the EEG starts to have periods of isoelectric signal, known as burst suppression periods, which in the limit with increasing drug concentrations cause a complete isoelectrical EEG. This is considered the deepest DoA level and is usually avoided.

It would be difficult or impossible for a human to process the EEG in real time. Automation of EEG processing is being explored by extracting features that correlate with the hypnotic state of the patient. The analysis can be made in the time, frequency, or time-frequency domains. The features that are usually extracted from the EEG signal are related to the amplitude, the power of the signal, or the power density distribution in the frequency or time domain, combined with the percentage of burst suppression. The aim is to obtain an estimate of the prob- ability density functions (PDFs) of variables that are relevant to assessing the hypnotic state of the patient and their changes over time. Histograms, Fourier analysis, wavelets, and bispectral (BIS) analysis are mathematical tools for generating PDFs of features (or subparameters). These features are weighted according to correlation factors that are obtained from databases of controlled clinical studies to produce a normalization rule that yields a number between zero and 100 that describes the DoA level, where zero represents the isoelectric EEG and 100 represents a patient in the awake state.

EEG signals can also be characterizing in terms of an entropy index, which is linked with the notion of complexity associated with predictable/unpredictable or regular/irregular properties of the EEG signal. This concept has its roots in the idea of Shannon's entropy. Other entropy types have been proposed, such as approximate, spectral, Tsallis, Kolmogorov, and time-frequency balanced spectral entropy. The entropy/Datex-Ohmeda index is based on spectral entropy, where the discrete Fourier transform is applied to EEG epochs to obtain the power spectrum. The power spectrum is then normalized and used to compute the Shannon entropy. An interpretation of this approach is that the message is composed by symbols defined by the frequency content of the EEG that are associated with the hypnotic state of the patient. Additional details can be found in [S9].

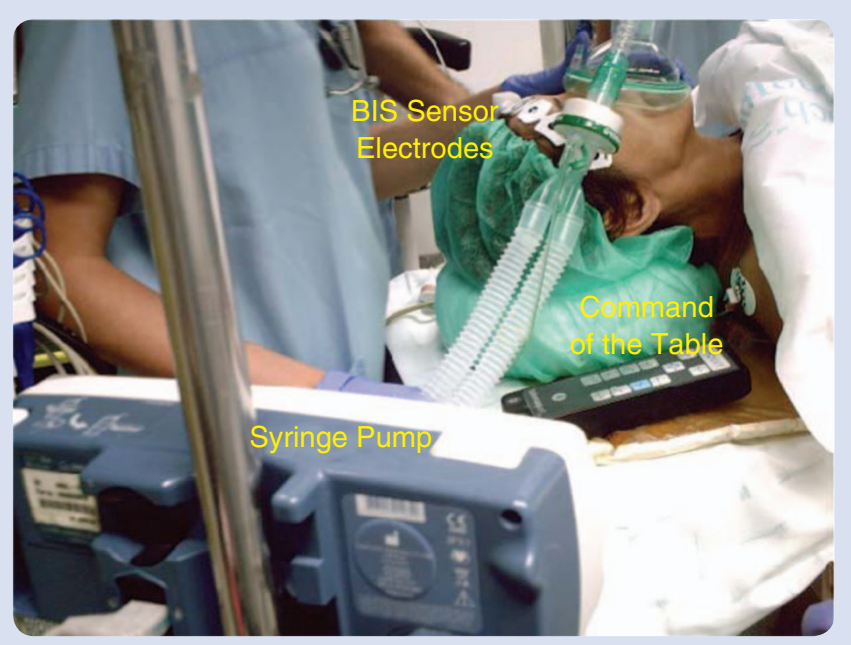

FIGURE S3 The patient in the operating room, in the initial phase of the anesthesia with the anesthetist holding the mask that supplies oxygen to the patient. The depth of anesthesia (hypnosis) is measured using the bispectral (BIS) sensor; the BIS electrodes are placed on the forehead of the patient. manipulated variables with the intermediate variables that are of main significance, such as the drug plasma concentration and the drug effect concentration (that is to say, the concentration at the place where the drug produces effect) and with the level of the desired index (such as NMB or BIS). For instance, the translation of neuronal electrical signals into muscle activity involves many electrochemical processes that interact in a chain to produce muscle contraction. These processes are affected by the presence of neuromuscular-blocking drug molecules in the cleft between the neuron and the muscle. Although it is possible to model all the above electrochemical reactions [29], [30], the resulting model would be 


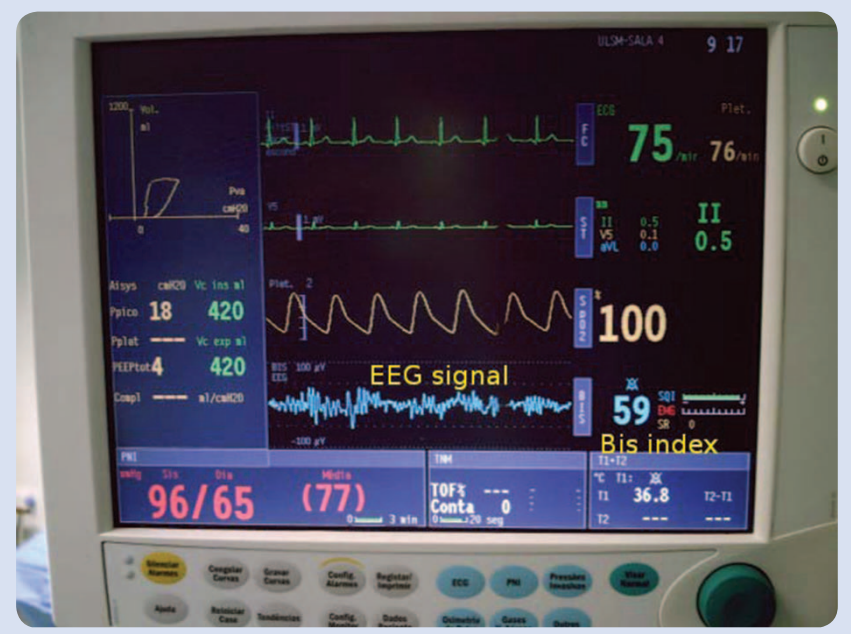

FIGURE S4 A view of the monitor with the information on the patient vital variables. Observe in particular the EEG signal during the anesthesia with the bispectral index indicating the value of 59 .

This article uses the BIS index implemented in the DatexOhmeda Entropy Module [S10]. Figure S3 shows the placement of the BIS sensor electrodes on the head of the patient, and Figure $S 4$ shows the monitor indicating the current value of the BIS index and part of the EEG signal. The BIS index has several versions that correspond to the evolution of the algorithm. Its basic implementation comprises several time- and frequency-domain (sub)parameters that are combined to form the index. The EEG is filtered to remove known artifacts, such as the mains power frequency, and then is split into time segments called epochs that are analyzed for the presence of additional artifacts to be removed. Additional tests are performed to validate the quality of the EEG epochs, for example, if they are corrupted by the electrical interference caused by the operation of an electrical bistoury or if they have eye blink signatures. Assuming that EEG epochs are free of artifacts, they are used to compute time- and frequency-domain parameters.

In the time domain, the burst suppression ratio (BSR) is computed, and an additional algorithm is used for robust detection of burst suppression in the presence of changes on the EEG baseline voltage. In the frequency domain, the fast Fourier transform is applied and the beta ratio is computed, which is the log power ratio between two empirically derived frequency bands, defined as $\log \left(P_{30-47 \mathrm{~Hz}} / P_{11-20 \mathrm{~Hz}}\right)$.

Another parameter, SynchFastSlow, is computed based on the analysis of a bispectrum, which quantifies the relationship between different sinusoidal components of the EEG. The aim is to evaluate the coupling between the components of two primary frequencies, $f_{1}$ and $f_{2}$, and a modulation component of frequency $f_{1}+f_{2}$. The bispectrum $B\left(f_{1}, f_{2}\right)=X\left(f_{1}\right) X\left(f_{2}\right) \bar{X}\left(f_{1}+f_{2}\right)$ incorporates both phase and power information, where $X\left(f_{i}\right)$ is the complex spectral value at frequency $f_{i}$ and $\bar{X}$ is the complex conjugate of $X$. SynchFastSlow is the log of the ratio of the sum of all bispectrum peaks in a frequency range from 0.5 to $47 \mathrm{~Hz}$ to the sum of all bispectrum peaks in a frequency range from 40 to $47 \mathrm{~Hz}$ [S10], [S11]. The BIS index is a weighted combination of these parameters, with weights determined by regression of data collected for many patients.

The above measure does not take into account phase information in the bispectrum, as the physiological meaning of phase coupling is uncertain [S10] and according to [S12], "the traditional explanation of the bispectrum as a measure of interfrequency phase coupling is misleading when applied to most real EEG signals." But according to [S13], "EEG bicoherence was sensitive to noxious stimuli, which suggested that EEG bicoherence would become an indicator for adequacy of analgesia during anesthesia." These two works show that there are some aspects of how to define the most clinically relevant bispectral index are not fully understood and that how to best assess the loss and recovery of consciousness is still an open research problem [S14].

\section{REFERENCES}

[S8] L. C. Jameson and T. Sloan, "Using EEG to monitor drug effects during surgery," J. Clin. Monit. Comput., vol. 20, pp. 445-472, Dec. 2006. [S9] H. Viertiö-Oja, V. Maja, M. Särkelä, P. Talja, N. Tenkanen, H. Tolvanen-Laakso, M. Paloheimo, A. Vakkuri, A. Yli-Hankala, and P. Meriläinen, "Description of the Entropy ${ }^{\mathrm{TM}}$ algorithm as applied in the Datex-Ohmeda $\mathrm{S} / 5^{\mathrm{TM}}$ entropy module," Acta Anaesthesiol. Scand., vol. 48, no. 2, pp. 154-161, 2004.

[S10] I. J. Rampil, "A primer for EEG signal processing in anesthesia," Anesthesiology, vol. 89, no. 4, pp. 980-1002, 1998.

[S11] J. C. Sigl and N. G. Chamoun, "An introduction to bispectral analysis for the electroencephalogram," J. Clin. Monit. Comput., vol. 10, pp. 392-404, Nov. 1994.

[S12] A. Miller, J. W. Sleigh, J. Barnard, and D. A. Steyn-Ross, "Does bispectral analysis of the electroencephalogram add anything but complexity?" Br. J. Anaesth., vol. 92, pp. 8-13, Jan. 2004.

[S13] S. Hagihira, M. Takashina, T. Mori, H. Ueyama, and T. Mashimo, "What we can know from bispectral analysis of EEG?" Int. Congr. Ser., vol. 1283, pp. 239-242, Nov. 2005.

[S14] P. L. Purdon, E. T. Pierce, E. A. Mukamel, M. J. Prerau, J. L. Walsh, K. F. K. Wong, A. F. Salazar-Gomez, P. G. Harrell, A. L. Sampson, A. Cimenser, S. Ching, N. J. Kopell, C. Tavares-Stoeckel, K. Habeeb, R. Merhar, and E. N. Brown, "Electroencephalogram signatures of loss and recovery of consciousness from propofol," in Proc. Nat. Academy Sciences, Mar. 2013, vol. 110, pp. E1142-E1151. too complex and would depend on too many parameters to estimate from the data available in clinical practice to be useful for control purposes.

To obtain a simpler model, aggregate models are considered by neglecting the dynamics of fast subsystems. According to the approach of compartmental model theory
[31], the body is divided into a (reduced) number of compartments, which correspond to "reservoirs" that exchange drugs among them. In NMB, one reservoir is the plasma and another is the cleft, called the "effect compartment" because the effect of the drug depends on its concentration in the cleft. Writing the balance equations by taking into 


\section{Neuromuscular Blockade and Hypnotic Models}

Trat

he effect of the both muscular relaxant and hypnotic drugs (in

this case, rocuronium and propofol, respectively) can be modeled by multicompartment models that comprise both pharmacokinetics (PK) and phamacodynamic (PD) models. These models have the structure of a linear model in series with a static nonlinearity modeled by the Hill equation described below. The effect of muscula-relaxant drugs on the patient [S15], [S16] is modeled by a three-compartment PK model, as shown is Figure S5, and the effect of the hypnotic drug [S17]-[S19] is modeled by the fourcompartment PK model shown in Figure S6.

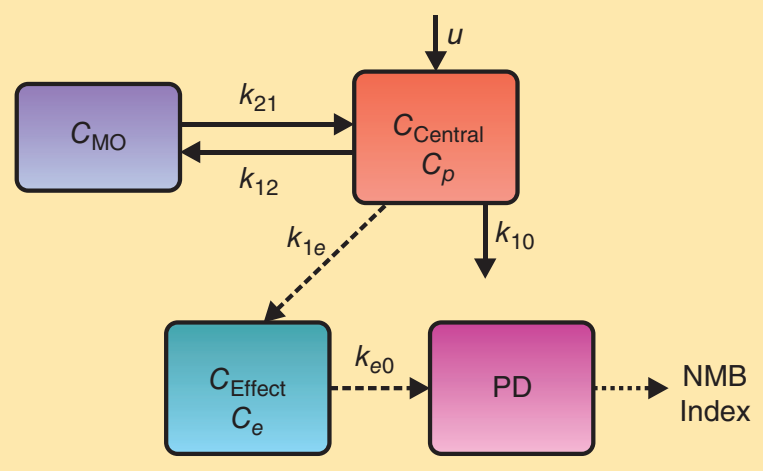

FIGURE S5 A schematic representation of the multicompartmental model for the neuromuscular blockade dynamic response. The nonlinear Hill equation is denoted as PD (for "pharmacodynamic"). The linear model is assumed to be made of three compartments that interchange drug due to interactions among them. These compartments are $C_{\text {central }}$ consisting mainly of the blood and liver, $C_{\text {Mo }}$ consisting mainly of muscle and organs, and $C_{\text {effect }}$ associated with the drug effect. The drug concentration in $C_{\text {central }}$ is denoted $c_{p}$ for "plasma concentration." This concentration results from the influx of the drug, with flow $u$, the drug efflux to compartment $C_{\text {MO }}$ given by $k_{12}\left(c_{p}-C_{\text {MO }}\right)$, the influx from compartment $C_{\mathrm{MO}}$ to compartment $C_{\text {central }}$ given by $k_{21}\left(c_{\mathrm{MO}}-c_{p}\right)$, the drug elimination efflux $k_{10} c_{p}$, and the efflux to the effect compartment $C_{\text {effect }}$ given by $k_{1 e} C_{p}$. In these rate expressions, the variable $c_{\text {MO }}$ denotes the drug concentration in compartment $C_{\text {MO }}$ and $C_{e}$ denotes the drug concentration in the effect compartment $C_{\text {effect }}$.
In both models, the drug is infused in the central compartment $C_{\text {central, }}$ which represents the blood, liver, and brain, and interacts with the other compartments. The drug is assumed to be uniformly distributed in each compartment. The compartment $C_{\mathrm{MO}}$ represents the drug distribution to the muscles and organs, and the compartment $C_{\mathrm{BF}}$ represents the drug distribution to the bone and fatty tissue. The interaction between these compartments relates the drug infusion rate $u$ with the drug concentration in the plasma, $c_{p}$, which, in turn, is related to the drug concentration in the effect compartment, $c_{e}$. The mathematical model that describes the drug-patient pharmacokinetics is written in state-space form as

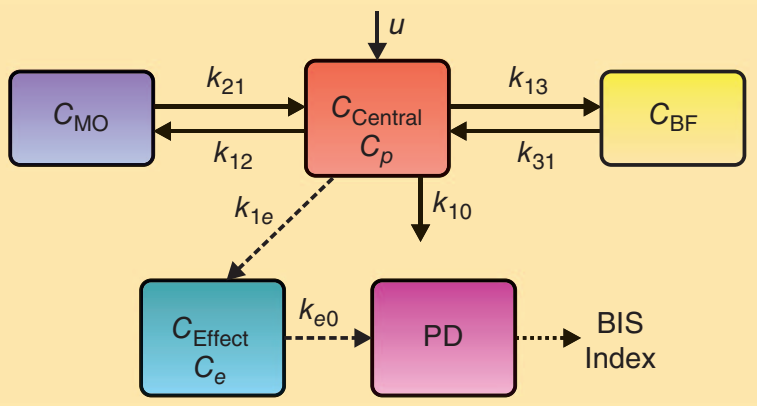

FIGURE S6 A schematic representation of the multicompartmental model for the hypnosis dynamic response. The nonlinear Hill equation is denoted as PD (pharmacodynamic). The linear model is a four-compartment model in which the central compartment $C_{\text {central }}$ is interconnected with compartments $C_{\mathrm{MO}}$ and $C_{\mathrm{BF}}$. Compartment $C_{\mathrm{BF}}$ consisting mainly of the bones and fatty tissue. The drug concentration in $C_{\text {central, }} c_{p}$, results from the influx of the drug, with flow $u$, the drug efflux from compartment $C_{\mathrm{MO}}$ given by $k_{12}\left(c_{p}-c_{\mathrm{MO}}\right)$, the influx from compartment $C_{\mathrm{MO}}$ to compartment $C_{\text {central }}$ given by $k_{21}\left(c_{\mathrm{MO}}-c_{p}\right)$, the drug efflux from compartment $C_{\mathrm{BF}}$ given by $k_{13}\left(C_{p}-C_{\mathrm{BF}}\right)$, the influx from compartment $C_{\mathrm{BF}}$ to compartment $C_{\text {central }}$ given by $k_{31}\left(c_{\mathrm{BF}}-c_{p}\right)$, the drug elimination efflux $k_{10} c_{p}$, and the efflux to the effect compartment $C_{\text {effect }}$ given by $k_{1 e} C_{p}$. In these rate expressions, the variable $c_{M O}$ denotes the drug concentration in compartment $C_{\mathrm{MO}}, C_{\mathrm{BF}}$ denotes the drug concentration in compartment $C_{\mathrm{BF}}$, and $C_{e}$ denotes the drug concentration in the effect compartment. account the drug interchange between the different reservoirs [31] yields a linear state-space model whose input is the drug infusion flow rate and whose states are the drug concentration in the different compartments.

The relation between the drug effect concentration and the NMB level is assumed to be given by a static nonlinear function called the "Hill equation." The Hill equation is not derived from first principles but is a good approximation to observed clinical data. For zero effect concentration, the Hill equation yields an NMB index of 100\% (see “Neuromuscular Blockade Monitoring and NMB Indices"), while for higher values the NMB index decreases. Figure 4 shows the overall structure of the NMB model together with a qualitative sketch of the time evolution of the main signals. Further details are given in "Neuromuscular Blockade and Hypnotic Models."

A similar approach can be followed with respect to DoA, resulting in the nonlinear model with the overall structure shown in Figure 5. The details are described in "Neuromuscular Blockade and Hypnotic Models." A major difference with respect to NMB is that, in this case, the DoA level is now affected by two drugs, namely the hypnotic drug and the analgesic drug. In mathematical terms, the 


$$
\left\{\begin{array}{l}
\dot{x}(t)=\Phi x(t)+\Gamma u(t) \\
C_{e}(t)=\mathbb{I} x(t)
\end{array}\right.
$$

where $\Phi$ is a patient-dependent matrix; $\Gamma$ is a column vector with the first element equal to $\Gamma_{1}$ and the remaining elements equal to zero, with $\Gamma_{1}=1$ for the NMB model and $\Gamma_{1}=16.7$ (a conversion factor) for the DoA model; II is a row vector with the last element equal to one and the remaining elements equal to zero; and $t$ is continuous time. For the NMB model, the variable $x(t)$ is the state whose entries are the drug concentrations in the different compartments. For the DoA model, the state is the mass in each compartment $\left(C_{\text {central }}, C_{\mathrm{MO}}\right.$, and $\left.C_{\mathrm{BF}}\right)$ and whose last element is $c_{e}$. The matrix $\Phi$ of the NMB model is

$$
\Phi=\left[\begin{array}{ccc}
-\left(k_{10}+k_{12}+k_{1 e}\right) & k_{21} & k_{31} \\
k_{12} & -k_{21} & 0 \\
k_{1 e} & 0 & -k_{e 0}
\end{array}\right],
$$

where $k_{i j}$ is the equilibrium constant between compartments. In particular, $k_{1 e}$ is the equilibrium constant between the central and the effect compartments and is assumed to be equal to $k_{e o}$. For the DoA model, the patient-dependent matrix $\Phi$ is

$$
\Phi=\left[\begin{array}{cccc}
-\left(k_{10}+k_{12}+k_{13}\right) & k_{21} & k_{31} & 0 \\
k_{12} & -k_{21} & 0 & 0 \\
k_{13} & 0 & -k_{31} & 0 \\
\frac{k_{1 e}}{1,000 V_{c}} & 0 & 0 & -k_{e o}
\end{array}\right],
$$

where $V_{c}$ is the volume of the $C_{\text {central }}$.

The nonlinear PD model of NMB, which relates the drug concentration in the effect compartment with the NMB index ( $T_{1}$ ratio), is given by the Hill equation

$$
\mathrm{NMB}=100 \frac{C_{50}^{\gamma}}{C_{50}^{\gamma}+C_{e}^{\gamma}}
$$

where $C_{50}$ and $\gamma$ are patient-dependent parameters. The parameter $C_{50}$ is interpreted as the value of the effect concentration that corresponds to the NMB index of $50 \%$. The NMB index is a normalized scale, in which $0 \%$ corresponds to full paralysis and $100 \%$ corresponding to no blockade effect at all.

For the hypnotic model, the drug effect observed on the patient is expressed as a nonlinear function of the drug concentration in the effect compartment, given by

$$
\mathrm{BIS}=E_{0}+\left(E_{\max }-E_{0}\right) \frac{C_{e}^{\gamma}}{C_{e}^{\gamma}+C_{50}^{\gamma}},
$$

where BIS is the BIS index of DoA, $E_{0}$ is the baseline effect at zero concentrations, $E_{\max }$ is the peak drug effect, $C_{50}$ is the concentration related with $50 \%$ of the drug effect, and $\gamma$ is the steepness of the concentration-response relation.

There is a synergetic effect between the analgesic drug remifentanil and the hypnotic drug propofol [S19] that is revealed in the electroencephalographic measure BIS index (see "Monitoring the Depth of Anesthesia (DoA)" for further information on hypnosis indices). This synergic relationship can be expressed in the overall effect as

$$
\mathrm{BIS}=\frac{97.7}{1+\left((1+\beta) U^{\text {prop }}+U^{\text {remi }}\right)^{\gamma}},
$$

where $\beta$ is a patient-dependent parameter and $U^{\text {prop }}$ and $U^{\text {remi }}$ are the normalized effect concentrations defined as

$$
U^{\text {prop }}=\frac{C_{e}^{\text {prop }}}{C_{50}^{\text {prop }}}, \quad U^{\text {remi }}=\frac{C_{e}^{\text {remi }}}{C_{50}^{\text {remi }}},
$$

where the superscript prop refers to the variables associated with propofol and the hypnotic model, whereas the superscript remi refers to the variables associated with remifentanil and the analgesic model. In this article, the remifentanil dose appears in the model as a disturbance. Although knowing its value can be taken as an advantage, a feedforward term is not considered in this article.

\section{REFERENCES}

[S15] B. C. Weatherley, S. G. Williams, and E. A. Neill, "Pharmacokinetics, phamacodynamics and dose-response relationships of atracurium administered i.v.," Br. J. Anaesth., vol. 55, no. 1, pp. 39S-45S, 1983. [S16] S. Ward, E. A. Neill, B. C. Weatherley, and I. M. Coral, "Pharmacokinetics of atracurium besylate in healthy patients (after a single i.v. bolus dose)," Br. J. Anaesth., vol. 55, no. 2, pp. 113-118, 1983.

[S17] B. Marsh, M. White, N. Morton, and G. N. C. Kenny, "Pharmacokinetic model driven infusion of propofol in children," Br. J. Anaesth., vol. 67, pp. 41-48, July 1991.

[S18] T. W. Schnider, C. F. Minto, S. L. Shafer, P. L. Gambus, C. Andresen, D. B. Goodale, and E. J. Youngs, "The influence of age on propofol pharmacodynamics," Anesthesiology, vol. 90, pp. 1502-1516, June 1999. [S19] T. W. Bouillon, J. Bruhn, L. Radulescu, C. Andresen, T. J. Shafer, C. Cohane, and S. L. Shafter, "Pharmacodynamic interaction between propofol and remifentanil regarding hypnosis, tolerance of laryngoscopy, bispectral index, and electroencephalographic approximate entropy," Anesthesiology, vol. 100, pp. 1353-1372, June 2004. interaction between both classes of drugs is represented by the Hill equation modified to have two inputs.

The control of NMB and DoA both rely on indices computed from online physiological data. As such, what is actually controlled are the values of the indices. Using different indices would lead to different results, and it is still a matter of debate among the scientific community what are the best indices. In "Neuromuscular Blockade Monitoring and NMB Indices" and in "Monitoring the Depth of Anesthesia (DoA)," the indices are explained in general terms.
For DoA, the BIS index is used, which takes its name from the fact that it relies on the bispectrum of the electroencephalogram. In this article, "BIS" is used in some formulas to refer to this index.

At the beginning of the anesthesia (during the induction phase), the anesthetist applies a drug bolus to the patient to drive the corresponding index close to the desired value. This fast procedure is subject to clinical constraints for the sake of patient safety. It is only then that the automatic controller starts to operate, for a period that may last several hours in 


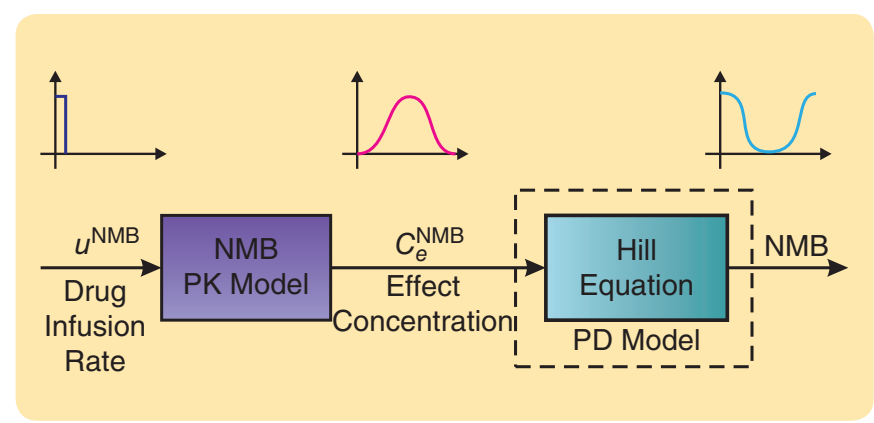

FIGURE 4 The overall structure of the nonlinear neuromuscular blockade (NMB) model. Mathematical models of the different blocks are given in "Neuromuscular Blockade and Hypnotic Models." The model comprises a linear dynamic part, called the pharmacokinetic (PK) model, that relates the drug infusion rate to the plasma and the effect concentrations, and a static nonlinear part described by the Hill equation called the pharmacodynamic (PD) model, that describes the relation between the effect concentration and the NMB level. In simplistic terms, the pharmacokinetic model is sometimes said to describe "what the body does to the drug" because it describes the drug propagation inside the body, from compartment to compartment, while the pharmacodynamic model is said to describe "what the drug does to the body" because it relates the drug concentration with its observed effect. The NMB model is a Wiener-type model, made of a dynamic linear model in series with a nonlinear static function. This figure also shows the typical time evolution of the variables in response to a bolus of drug. The NMB index $T_{1}$, that is initially at $100 \%$, decreases with the increase of drug concentration in the effect compartment, to increase again when the drug disappears.

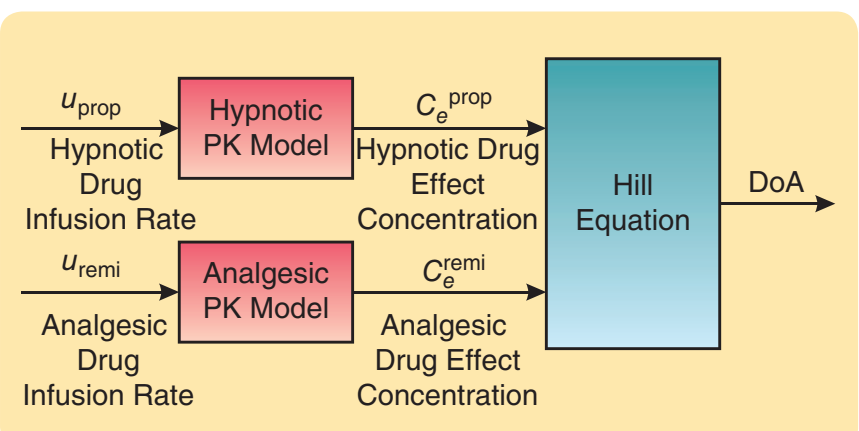

FIGURE 5 The overall structure of the nonlinear depth of anesthesia (DoA) model. Mathematical models of the different blocks are given in "Neuromuscular Blockade and Hypnotic Models." The structure is similar to that of the neuromuscular blockade (NMB) model, with the main difference being that there are now two drugs affecting the output. In contrast to the NMB model, the Hill equation now has two inputs that are combined algebraically to yield the DoA index. In this article, the hypnotic drug infusion rate is the manipulated variable, and the analgesic drug infusion rate is considered to be a disturbance.

the maintenance phase. The aim is therefore to regulate the anesthesia indices around references that are constant most of the time. Given this fact, linearized models, using Jacobian linearization, are considered for NMB and DoA. Since both these systems are of Wiener type (see "Neuromuscular Blockade and Hypnotic Models" for a detailed description of the nonlinear models), their Jacobian linearization simply amounts to replacing their static output nonlinearities (defined by the Hill equation) with a gain. Assuming some uncertainty on this gain allows the controller to work for values of the reference that differ from the nominal value.

\section{UNCERTAINTY CHARACTERIZATION}

The patient's dynamic response to the drug infusion rate is highly dependent on the patient's characteristics, such as age, gender, and weight, as well as on the drug used. The response also depends on the patient's genetic characteristics. This fact generates high levels of uncertainty when modeling the patient's dynamic response. To characterize the dynamics, two patient model databases are used. For the NMB model, a database of 50 patient models denoted $\mathcal{G}_{\mathrm{NMB}}=\left\{G_{i}^{\mathrm{NMB}}, i=1, \ldots, 50\right\}$ is considered, whereas a database of 18 models denoted $\mathcal{G}_{\mathrm{DOA}}=\left\{G_{i}^{\mathrm{DOA}}, i=1, \ldots, 18\right\}$ is considered for the DoA models. These databases have been obtained using system identification and statistical methods from primary patient data, for patients undergoing elective surgery at two hospitals in Portugal. The physical status classification of the patients, according to the American Society of Anesthesiologists (ASA), is ASA II and III. The degrees ASA are used by anesthesiologists to stratify the severity of patient's disease and potential for suffering complications from general anesthesia. The degree II refers to patients with mild systemic disease and no functional limitation, while degree III refers to patients with severe systemic disease and definite functional impairment. The main limitation of these two databases concerns other ASA degrees, in particular for patients with more severe conditions, so this case study does not allow any conclusions to be made on the effectiveness of the proposed control system for such patients.

For an input bolus of drug represented by a square function with very short duration and very large amplitude, Figures 6 and 7 show the simulated response for NMB and DoA, using the nonlinear model, for the nonlinear patient models in the databases. After the bolus is administered, the index drops initially and subsequently rises as the drug is metabolized to regain the initial value of $100 \%$. When control is used, the return to the $100 \%$ level is prevented by further administration of drug. The time responses shown in Figures 6 and 7 provide a qualitative indication of the uncertainty associated with patient-to-patient variability.

The first step in the control design is to define nominal models, which assume the form of continuous-time transfer functions

$$
G_{N}^{\text {DoA }}(s)=\frac{-0.0317 s^{2}-2.373 \times 10^{-3} s-7.943 \times 10^{-6}}{s^{4}+1.105 s^{3}+0.323 s^{2}+0.0124 s+1.836 \times 10^{-5}}
$$

for DoA (using the BIS index), assuming a nominal reference of BIS $=50$, and

$$
G_{N}^{\mathrm{NMB}}(s)=\frac{-0.1705 s-1.827 \times 10^{-3}}{s^{3}+0.2019 s^{2}+9.831 \times 10^{-3} s+5.89 \times 10^{-5}},
$$

for $\mathrm{NMB}$, with a nominal reference of $10 \%$. 


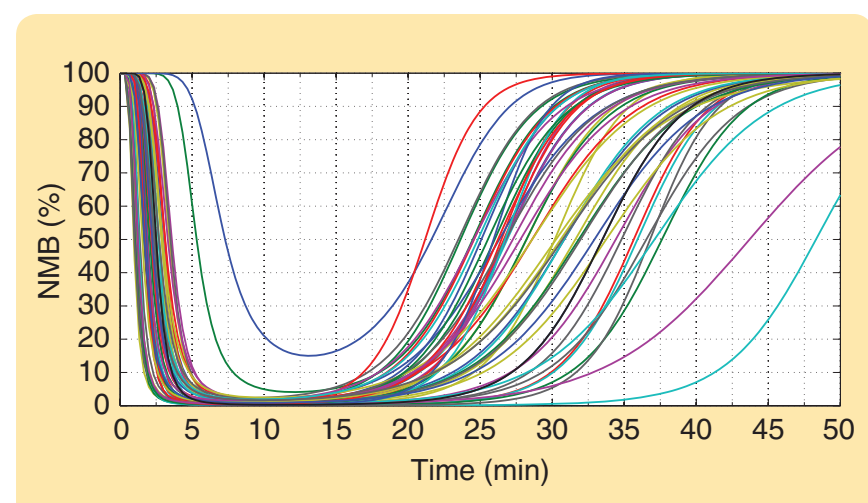

FIGURE 6 Neuromuscular blockade (NMB) simulated responses to a bolus of rocuronium for the patient models in $G_{\mathrm{NMB}}$.

Figure 8 shows the frequency response plots of $G_{N}^{\text {DoA }}$ and the models in the database for DoA. Similar plots have been also obtained for NMB (these plots are omitted for simplicity). As seen from Figure 8, the nominal model is of low-pass type, with a low frequency phase of $180^{\circ}$ that reflects the fact that an increase of the drug dosage leads to a decrease of the output index.

Multiplicative uncertainty is computed from the nominal model $G_{N}^{x x x}$ and the set of database models $G_{i}^{x x x}$. The normalized distance between each model in the database and the nominal model at frequency $\omega$ is

$$
\left|\Delta_{i}^{x x x}(j \omega)\right|=\left|\frac{G_{N}^{x x x}(j \omega)-G_{i}^{x x x}(j \omega)}{G_{N}^{x x x}(j \omega)}\right|,
$$

where $x x x$ is either NMB or DoA, and $i=1, \ldots, 50$ for NMB models and $i=1, \ldots, 18$ for DoA models.

An upper-bound $l_{x x x}(\omega)$ on the normalized distance $\left|\Delta_{i}^{x x x}(j \omega)\right|$ is used to build a multiplicative description of the uncertainty. The upper bound is over all models in the class. This bound, together with the error function $\left|\Delta_{i}^{\mathrm{DoA}}(j \omega)\right|$, is shown in Figure 9 (plotted in red), for DoA. From this figure, it is apparent that the bound on the multiplicative uncertainty is a high-pass function, being smaller for frequencies up to $0.2 \mathrm{rad} / \mathrm{min}$. Similar plots have been obtained for NMB but are omitted for the sake of brevity.

The characterization of the model, and of the respective uncertainty, consists of the nominal models $G_{N}^{\text {DoA }}$ for DoA and $G_{N}^{\mathrm{NMB}}$ for $\mathrm{NMB}$, together with the multiplicative uncertainty bounds $l_{\mathrm{DoA}}(\omega)$ and $l_{\mathrm{NMB}}(\omega)$. These descriptions are used below to design robust controllers for NMB and DoA.

\section{CONTROLLER DESIGN}

Figure 10 shows a schematic representation of the interconnection of the controller $K$ with the process $G$ (either DoA or NMB). An integrator is embedded in $K$ to ensure zero steadystate error when tracking constant references. The manipulated input is $u$, the measured output is $y, n$ is sensor noise, and $d$ is the output disturbance. The closed-loop objectives are specified by the selection of the transfer functions $W_{S}$ and $W_{T}$, with

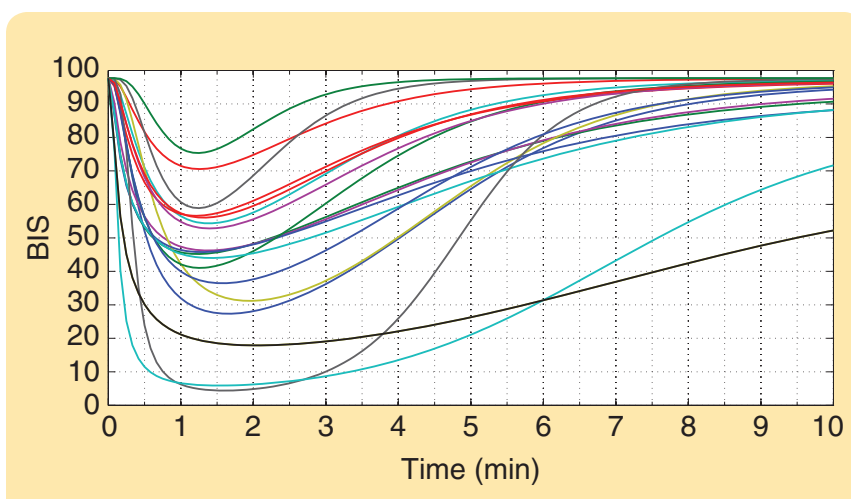

FIGURE 7 Depth of anesthesia simulated responses to a bolus of propofol for the patient models in $G_{\text {DoA }}$.

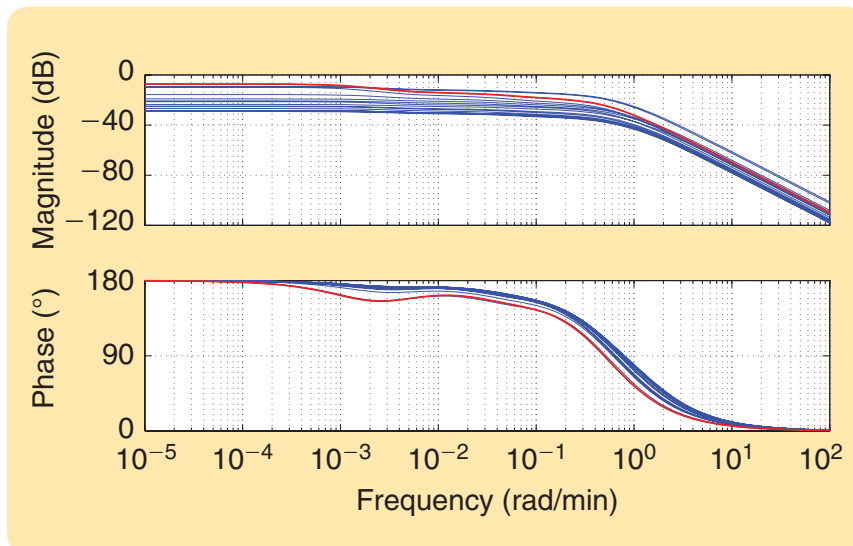

FIGURE 8 Frequency responses of the patient models in the neuromuscular blockade model database: a comparison between the nominal model (in red) and the remaining models in the database (in blue).

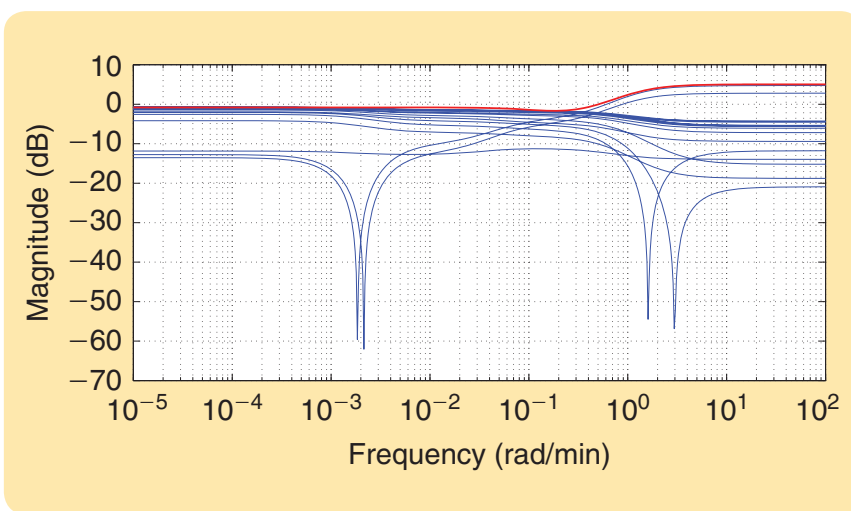

FIGURE 9 The multiplicative uncertainty of the patient models in the depth of anesthesia model database. The multiplicative uncertainty of the models with respect to the nominal model (in blue) is quantified by the upper bound function in red.

$W_{S}$ associated with performance (both setpoint tracking and suppression of the effects of disturbances) and $W_{T}$ associated with robustness to model uncertainties and insensitivity to measurement noise. To have low steady-state error while having a fast closed-loop response, the function $\left(W_{S}^{x x x}\right)^{-1}$ is chosen to have small gain at the low frequencies and high crossover frequency. On the other hand, the crossover frequency and 


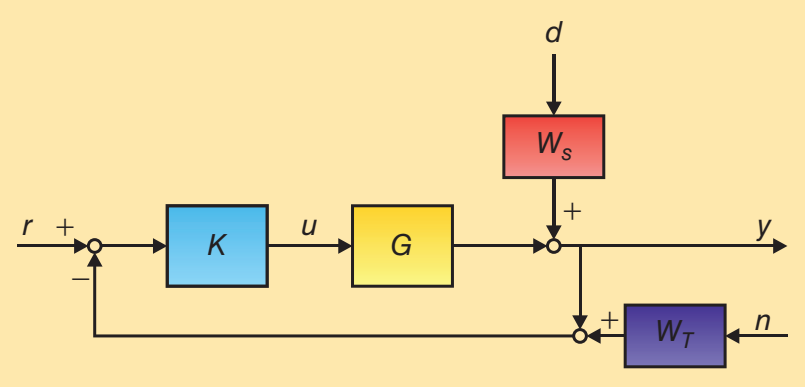

FIGURE 10 A schematic representation of the control system. The controller $K$ is designed with the $H_{\infty}$ approach to compute the input $u$ required for the system $G$ to track the desired output $r$. Here, the transfer function $W_{T}$ is designed to filter the effects of sensor noise $n$ and the transfer function $W_{S}$ is designed to suppress the effects of disturbances $d$ and for setpoint tracking. The structure is the same for both neuromuscular blockade and depth of anesthesia.

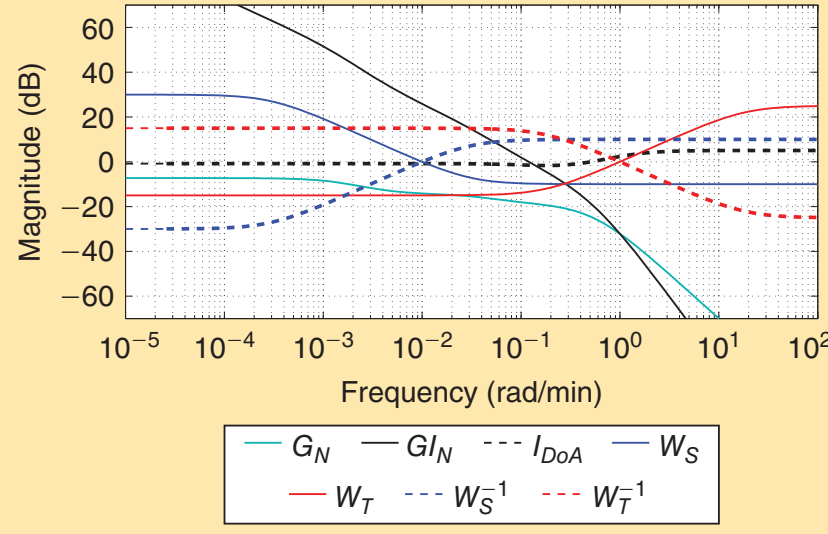

FIGURE 11 The depth of anesthesia robust control design with Bode magnitude plots of $W_{S}^{\text {DoA }}$ and $W_{T}^{\text {DoA }}$ and of the respective inverses $\left(W_{S}^{\mathrm{DOA}}\right)^{-1}$ and $\left(W_{T}^{\mathrm{D} O A}\right)^{-1}$. The nominal model $G_{N}^{\mathrm{DoA}}$ and the upper bound $I_{\text {DoA }}$ are also shown. The curve $G I_{N}$ is the nominal model in series with the integrator incorporated into the controller.

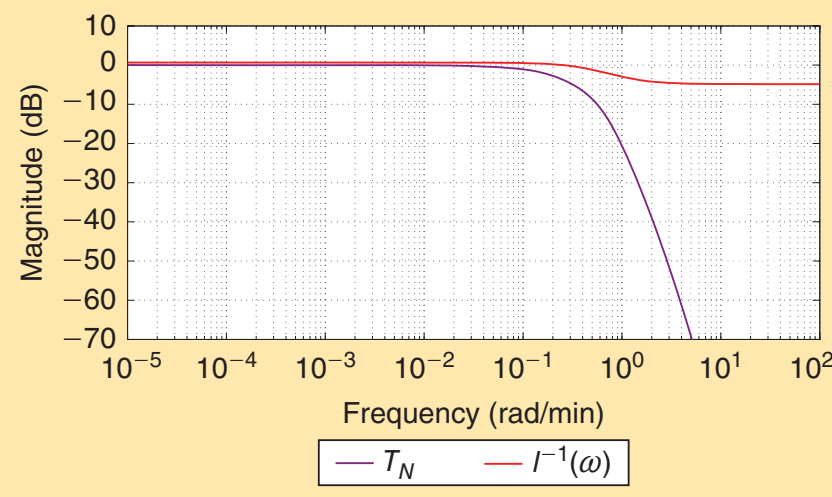

FIGURE 12 The depth of anesthesia control design and the robust stability condition. The Bode magnitude plot of the nominal complementary sensitivity $T_{N}^{\mathrm{DoA}}$ and the inverse of the upper bound $I_{\mathrm{DoA}}(\omega)$. This plot indicates that the robust stability condition (4) is satisfied. the high frequency gain of the functions $\left(W_{T}^{x x x}\right)^{-1}$ must be low enough to ensure adequate insensitivity to high frequency noise and model uncertainty [27]. To ensure robust stability to the specific uncertainties quantified in the previous section, the controller (for DoA and NMB) must satisfy

$$
\frac{1}{l_{x x x}(\omega)}>\left|T_{N}^{x x x}(j \omega)\right| \text {, for all real } \omega
$$

where $T_{N}^{x x x}(s) \triangleq K G_{N}^{x x x} / 1+K G_{N}^{x x x}$ is the complementary sensitivity for the nominal plant.

Most applications apply $H_{\infty}$ design conditions only to the nominal plant; here we also applied the conditions to all of the data models. For either DoA or NMB, the sensitivity for the $i$ th database model is defined by

$$
S_{i}^{x x x} \triangleq \frac{1}{1+K G_{i}^{x x}}
$$

and the associated complementary sensitivity is defined by

$$
T_{i}^{x x x} \triangleq \frac{K G_{i}^{x x x}}{1+K G_{i}^{x x x}} .
$$

The controller $K$ was designed to satisfy the commonly used performance and robust stability conditions for all of the database models:

$$
\left|S_{i}^{x x x}(j \omega)\right|<\frac{1}{\left|W_{S}^{x x x}(j \omega)\right|} \text {, for all } i \text { and real } \omega,
$$

and

$$
\left|T_{i}^{x x x}(j \omega)\right|<\frac{1}{\left|W_{T}^{x x}(j \omega)\right|} \text {, for all } i \text { and real } \omega \text {. }
$$

This approach ensures a higher degree of robustness to model uncertainties than using the normal conditions that apply the inequalities only to the nominal plant. An alternative tuning method would have been to modify $W_{T}$ to have larger amount of model uncertainty.

The Bode magnitude plots for $\left(W_{S}^{\mathrm{DoA}}\right)^{-1}$ and $\left(W_{T}^{\mathrm{DoA}}\right)^{-1}$ shown in Figure 11 are typical of $H_{\infty}$-control design. The transfer function $\left(W_{S}^{\mathrm{DoA}}\right)^{-1}$ was defined to have a crossover frequency of $0.01 \mathrm{rad} / \mathrm{min}$, and the transfer function $\left(W_{T}^{\mathrm{DoA}}\right)^{-1}$ was selected to have a crossover frequency of $1 \mathrm{rad} / \mathrm{min}$. The former crossover frequency ensures a fairly fast closed-loop response speed, and the latter crossover frequency should not be larger as that would lead to poor suppression of high frequency noise. The gain of $\left(W_{S}^{\mathrm{DoA}}\right)^{-1}$ at low frequencies could not be made smaller while still satisfying (7).

The Matlab Robust Control Toolbox function $d k s y n$ was used to design a controller based on $H_{\infty}$ optimization. Figure 12 shows that the DoA controller fulfills the robust stability condition (4), and Figure 13 demonstrates that the robustness conditions (7) and (8) are satisfied.

The resulting controller has 19 states, which was approximated by a seventh-order controller. The controller order 


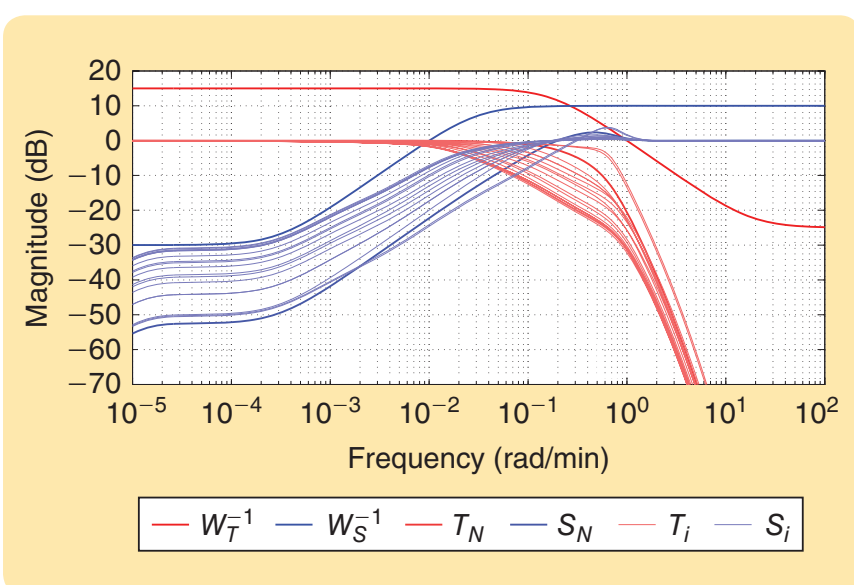

FIGURE 13 The depth of anesthesia robust control design. Bode magnitude plots of the sensitivity $S_{i}^{\text {DoA }}$ and the complementary sensitivity $T_{i}^{\mathrm{DoA}}$ for all of the database models, with the upper bounds $\left(W_{S}^{\text {DoA }}\right)^{-1}$ and $\left(W_{T}^{\text {DoA }}\right)^{-1}$. This plot shows that the robustness conditions (7)-(8) are fulfilled since the $W_{S}^{-1}$ and $W_{T}^{-1}$ are upper bounds of the functions $S_{i}^{\mathrm{DoA}}$ and $T_{i}^{\mathrm{DoA}}$, respectively.

reduction was performed by a balanced model truncation via the square root method [32]. An existing Matlab function was used for this purpose. For computer implementation, the DoA controller was discretized by the zero-order hold method, with a sampling interval of $5 \mathrm{~s}$. The resulting discrete-time controller was then combined with the discrete-time integrator.

The control design procedure was the same for the NMB control design, so the detailed design plots are not shown here. All of the design conditions (4), (7), and (8) were also satisfied by the NMB controller, with the respective transfer functions $W_{S}^{\mathrm{NMB}}$ and $W_{T}^{\mathrm{NMB}}$

The control algorithm was implemented on a laptop computer (shown in Figure 3) on the GALENO platform, which was designed for the purpose of automating anesthesia. GALENO is a Matlab package, developed by the authors, that allows for the interface of control algorithms with the medical equipment. The controller computes the appropriate drug dosage, which is sent to the syringe pump that, in turn, delivers the drug to the patient. Figure 14 shows a screen of the GALENO platform with the designed controller being used in real-time mode.

\section{CLINICAL RESULTS}

Several clinical experiments have been performed with the controllers for DoA and NMB.

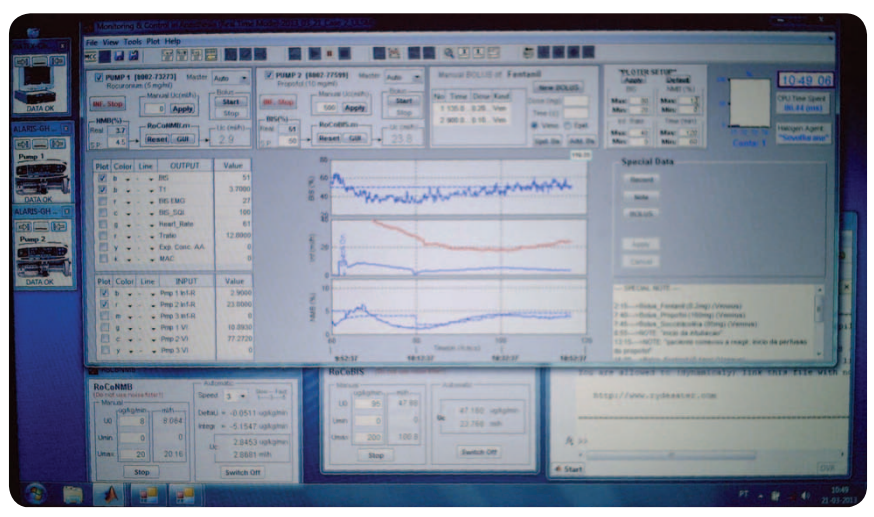

FIGURE 14 A computer display showing the GALENO anesthesia control system in operation, during one of the first tests in an early version of the platform. The display shows information concerning the control of neuromuscular blockade and depth of anesthesia, including the settings of the algorithm being used. In addition to tracking the reference, the associated operator interface allows changing between automatic (closed-loop) and manual control to administer to the patient a bolus of specified quantities of the relaxant or hypnotic drugs. The interface also allows including remarks-such as when the anesthetist manually administers a bolus of analgesic or when the electric bistoury is in operation-in the signal plots, which are useful for future interpretation of the data.

In the first case study presented, the DoA robust controller is used during a general anesthesia in a 56-year-old male patient, with a height of $1.50 \mathrm{~m}$ and a weight of $50 \mathrm{~kg}$, during a pancreatoduodenectomy (a surgery in which tumors are removed from the pancreas and surrounding organs). The results are shown in Figure 15. The patient is initially in a state characterized by a BIS index between 90 and 100 .

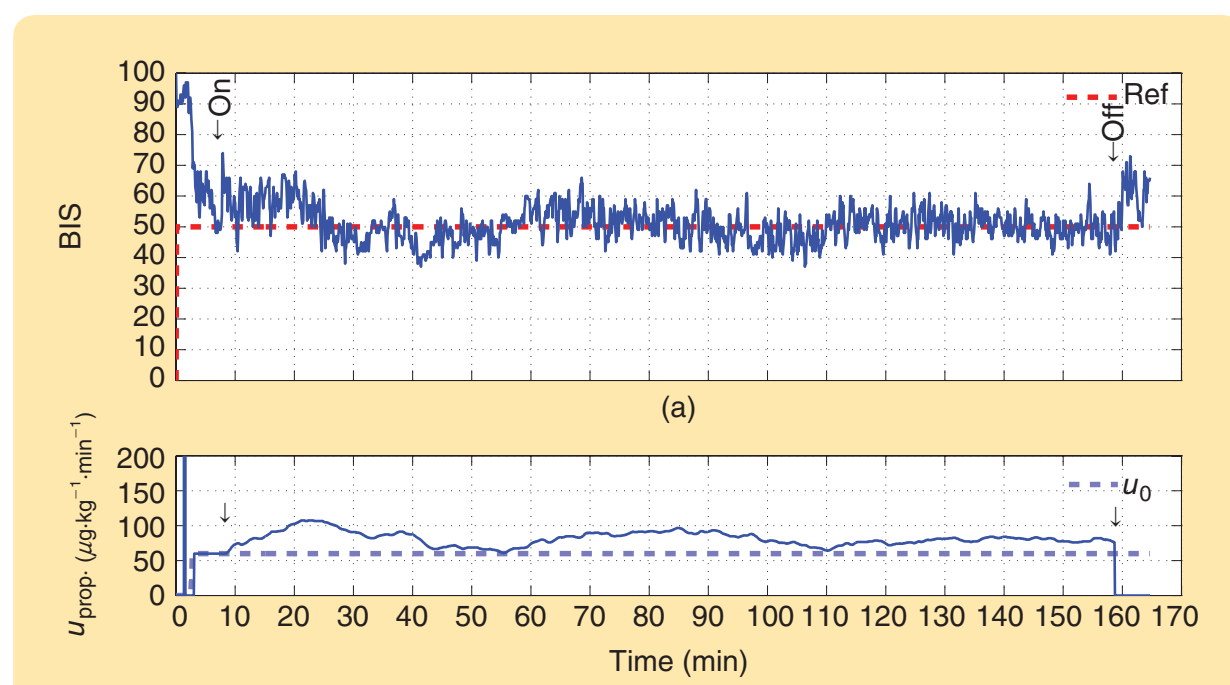

(b)

FIGURE 15 A clinical experiment: The patient response, during a general anesthesia, when the depth of anesthesia $H_{\infty}$ controller is used. The bispectral (BIS) index is plotted in blue in (a), with the reference level set to 50 (red dashed line) and the drug dose $u_{\text {prop }}$ delivered to the patient in (b). The arrows marked "On" and "Off" indicate when the controller is turned on and off, respectively. In the drug dose plot, the dashed line corresponds to the baseline equilibrium value of the drug dose, to which the incremental dose computed by the control algorithm is added. In some cases, the baseline value $u_{0}$ is adjusted manually for clinical convenience. In this figure, the value of $u_{0}$ is kept constant for the entire maintenance phase. 
A bolus of the hypnotic drug propofol is administered manually to the patient in the induction phase, driving his BIS index to around 60 in a few minutes. Subsequently, the pump is adjusted to provide a constant drug infusion that keeps the BIS index in equilibrium. These equilibrium values of the drug infusion and the average BIS index define an operating point around which the linear $H_{\infty}$ controller adjusts the drug infusion. When the controller is turned on at the time indicated by the vertical arrow $(9 \mathrm{~min})$, the rate of drug infusion is adjusted so that the BIS signal remains near the reference value of 50 . The whole intervention lasts about $2 \mathrm{~h}$ and $40 \mathrm{~min}$. Although the BIS signal is noisy, the controller is tuned to provide a relatively smooth manipulated variable. The controller is able to provide an adequate drug dosage during the entire procedure, maintaining the patient at the desired level of 50 .

In the second case study, both robust controllers for DoA and NMB are simultaneously put in operation during a general anesthesia in a 78-year-old male patient, with a height of $1.70 \mathrm{~m}$ and a weight of $60 \mathrm{~kg}$, during a hemicolectomy (an operation to remove a segment of the colon). The resulting drug dosage for both controllers are presented in Figure 16. In the case of DoA control, the controller computed the adequate amount of drug over the major part of the procedure. After the induction phase, the DoA controller is switched on. Since the controller was slow in driving the BIS index to the reference, the dose of the hypnotic drug was adjusted manually. After $t=40 \mathrm{~min}$, the controller is able to maintain the patient at the desired BIS level of 50 . The performance of the NMB controller is also appropriate during this surgery. After the induction phase, where a bolus is applied to induce a fast NMB-level drop to near $0 \%$ and after a manual manipulation of the drug dosage to stabilize the patient, the controller is switched on. During this maintenance phase, the controller is able to compute the adequate drug dosage to maintain the NMB level at the desired reference of $5 \%$.

The NMB controller has been compared to a pole-placement controller that was developed based on a trial-and-error process to guarantee robust stability. The controller has been designed by adjusting the time response specifications and verifying a posteriori the robust stability condition. Results with this controller are presented in Figure 17, where its performance is shown during general anesthesia on a 75-yearold male patient, with a height of $1.75 \mathrm{~m}$ and a weight of $68 \mathrm{~kg}$, during a cephalic pancreatoduodenectomy. With the NMB pole-placement controller, the control action must be assisted by imposing a hard upper bound. This strategy avoids the aggressive control action and noise amplification that would otherwise probably lead to excessive infusion doses and a consequent drop of the NMB level to $0 \%$. This pole-placement controller has a poor closedloop performance compared to the $H_{\infty}$-based controller, which is able to filter high frequency sensor noise and deliver the adequate dosage.
FIGURE 16 A clinical experiment: The patient response during a general anesthesia, using both depth of anesthesia (DoA) and neuromuscular blockade (NMB) $H_{\infty}$ controllers. The arrow marked "On" indicates when the controller is turned on. The DoA level, measured by the bispectral (BIS) index, is represented in blue in (a), with desired level (red dashed line) and the drug dose $u_{\text {prop }}$ delivered to the patient in (b). The NMB level, measured by the $T_{1}$ ratio, is presented in

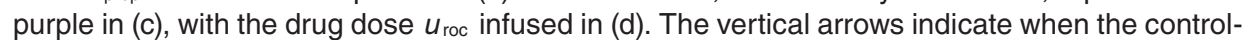
lers are switched on. The dashed lines in the BIS and $T_{1}$ plots represent the reference to track. 


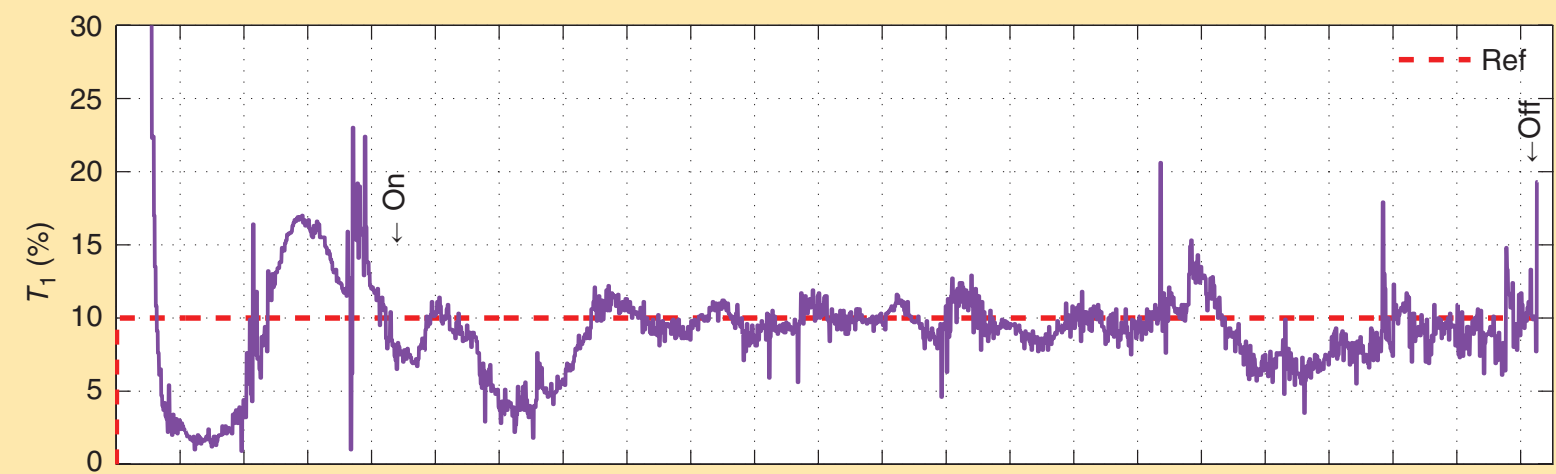

(a)

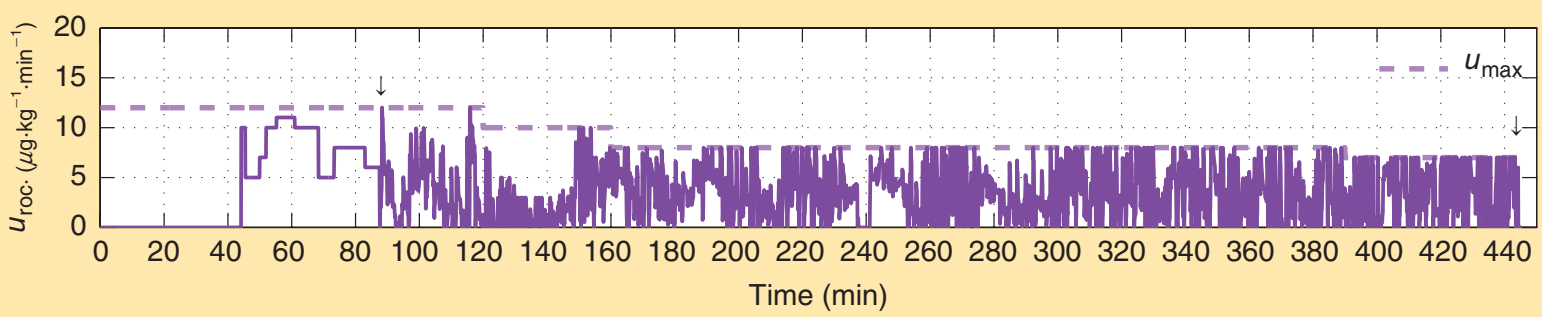

(b)

FIGURE 17 A clinical experiment: The patient response, during a general anesthesia, when the neuromuscular blockade (NMB) poleplacement controller, with robustness properties adjusted by trial and error, is used (a). The NMB level measured by the $T_{1}$ ratio is presented in purple and the desired level by the red dashed line. The drug dose $u_{\text {roc }}$ infused is shown in (b). The vertical arrows indicate when the controllers are switched on and off. When compared to $H_{\infty}$ control, the performance is poor, with the manipulated variable having more variation and often reaching saturation level $u_{\max }$ indicated by the dashed line.

\section{CONCLUSION}

This article demonstrates through clinical cases the use of a robust controller to regulate the NMB and hypnotic (BIS) indices during the maintenance phase of anesthesia applied to patients undergoing general elective surgery.

\section{ACKNOWLEDGMENT}

The work reported in this article was supported by FCT (Portugal) under contracts PTDC/SAU-BEB/103667/2008, PEst-OE/EEI/LA0021/2013, and PEst-C/MAT/UI4106/2011.

\section{AUTHOR INFORMATION}

João M. Lemos is a professor of systems, decision, and control in the Department of Electrical and Computer Engineering, Instituto Superior Técnico, Universidade de Lisboa, Portugal, and coordinator of the Control of Dynamic Systems research group at INESC-ID. His interests are concerned with applications of predictive, adaptive, and distributed control. Solar thermal systems, thermoelectric power plants, water delivery canals, and biomedical applications of control, including anesthesia and HIV-1 infection, are the main topics addressed.

DanielaV.Caiado (daniela.caiado@ist.utl.pt) received the M.S. degree in biological engineering from the University of Algarve, Faro, Portugal, in 2009. She is currently with the Control of Dynamic Systems research group at INESC-ID, Lisbon. Her research interests include mathematical modeling and control of biological systems. She can be contacted at Rua Aves Redol, 9, 1000-029 Lisboa, Portugal.
Bertinho A. Costa received the Ph.D. degree in electrical and computer engineering from the Instituto Superior Técnico (IST), Technical University of Lisbon, Portugal, in 1996. He is an assistant professor in the Department of Electrical and Computer Engineering, IST, and is a senior researcher of the research group on Control of Dynamic Systems at INESC-ID. His research interests include predictive, adaptive, and distributed control; model identification; programming; and realtime systems, with application to solar-energy systems, anesthesia, HIV-1 infection, cancer control, water delivery canals, and thermal power plants.

Luis $A$. Paz received the electronic engineering degree from the University of Pinar del Río, Cuba, in 1992, the M.Sc. degree in industrial control system from the University of Valladolid, Spain, in 2001, and the Ph.D. degree in chemical and biological engineering from the University of Porto, Portugal, in 2010. From 1992 to 2007 he worked as an associated researcher and auxiliary professor in the Department of Telecommunications and Electronics at the University of Pinar del Río, Cuba. He is currently a researcher in the GALENO project (PTDC/SAU-BEB/103667/2008) in the Department of Applied Mathematics at Faculdade de Ciências da Universidade do Porto. His research interests are in the area of computer control system, and he has been involved in projects to model and control industrial and biomedical systems.

Teresa F. Mendonça received the applied mathematics degree and the Ph.D. degree in applied mathematics (systems theory and signal processing) from Faculdade de Ciências da 
Universidade do Porto (FCUP), Portugal, in 1993. She is currently an assistant professor with the Department of Applied Mathematics at FCUP and a researcher with the Mathematical Systems Theory Group, UI \& D Matemática e Aplicações, Universidade de Aveiro, Portugal. Her research interests include control systems applied to biomedical systems. She has been involved in projects on modeling and control of anesthesia.

Rui Rabiço received the medicine degree from Faculdade de Medicina da Universidade do Porto, Portugal, in 2004. He completed his residency in anesthesiology in Hospital Pedro Hispano, Matosinhos, Portugal, in 2010 and is a diplomate of the European Society of Anaesthesiology. Currently he is a staff anesthesiologist at the Hospital Pedro Hispano Unidade Local de Saúde de Matosinhos and is involved in the clinical application of control for anesthesia.

Simão Esteves received the medicine degree from Instituto de Ciências Biomedicas Abel Salazar do Porto, Portugal, in 1982. He has been a consultant anesthesiologist with the Hospital de Santo António, Centro Hospitalar do Porto, since 1990. His special interests are in the field of pharmacology and monitoring of neuromuscular blockade. He has collaborated on projects dealing with the control of anesthesia and neuromuscular blockade for many years.

Manuel Seabra received the medical degree from the Faculdade de Medicina da Universidade do Porto in 1982. In 1990 he became a specialist in anesthesia and worked at the Serviço de Anestesia do Hospital de Santo António, in Porto, as an assistant in anesthesiology. In 1995 he moved to Serviço de Anestesia do Hospital Pedro Hispano, in Matosinhos, first as an assistant, and later on as a consultant in anesthesiology. He is currently head of the Department of Anesthesiology at the Hospital Pedro Hispano, Unidade Local de Sáude de Matosinhos. His research interests are in the area of control systems applied to anesthesia. He also supervises all the department clinical research.

\section{REFERENCES}

[1] M. Araki and E. Furutani, "Computer control of physiological states of patients under and after surgical operation," Annu. Rev. Control, vol. 29, no. 2, pp. 229-236, 2005.

[2] C. M. Wait, V. A. Goat, and C. E. Blogg, "Feedback control of neuromuscular blockade. A simple system for infusion of atracurium," Anaesthesia, vol. 42, no. 11, pp. 1212-1217, 1987.

[3] A. D. Macleod, A. J. Asbury, W. M. Gray, and D. A. Linkens, "Automatic control of neuromuscular block with atracurium," Br. J. Anaesth., vol. 63, pp. 31-35, July 1989.

[4] D. A. O’Hara, G. J. Derbyshire, F. J. Overdyk, D. K. Bogen, and B. E. Marshall, "Closed-loop infusion of atracurium with four different anesthetic techniques," Anesthesiology, vol. 74, pp. 258-263, Feb. 1991.

[5] T. Mendonça and P. Lago, "PID control strategies for the automatic control of neuromuscular blockade," Control Eng. Pract., vol. 6, no. 10, pp. 1225-1231, 1998 [6] M. Denai, D. A. Linkens, A. J. Asbury, A. D. MacLeod, and W. M. Gray, "Self-tuning PID control of atracurium-induced muscle relaxation in surgical patients," Inst. Elec. Eng. Proc. D Control Theory Applicat., vol. 137, pp. 261-272, Sept. 1990

[7] P. C. Uys, D. F. Morrell, H. S. Bradlow, and L. B. Rametti, “Self-tuning, microprocessor-based closed-loop control of atracurium-induced neuromuscular blockade," Br. J. Anaesth., vol. 61, pp. 685-692, Dec. 1988.

[8] A. Absalom, N. Sutcliffe, and G. N. Kenny, "Closed-loop control of anesthesia using bispectral index: Performance assessment in patients undergoing major orthopedic surgery under combined general and regional anesthesia," Anesthesiology, vol. 96, pp. 67-73, Jan. 2002.
[9] H. Schwilden, J. Schuttler, and G. Stoeckel, "Closed-loop control of methohexital anesthesia by quantitative EEG analysis in humans," Anesthesiology, vol. 67, no. 3, pp. 341-347, 1987.

[10] A. Gentilini, M. Rossoni-Gerosa, C. W. Frei, R. Wymann, M. Morari, M. Zbinden, and T. W. Schnider, "Modelling and closed-loop control of hypnosis by means of bispectral index (BIS) with isoflurane," IEEE Trans. Biomed. Eng., vol. 48, no. 8, pp. 874-889, 2001.

[11] M. Mahfouf, A. J. Asbury, and D. A. Linkens, "Unconstrained and constrained generalized predictive control of depth of anesthesia during surgery," Control Eng. Pract., vol. 11, no. 12, pp. 1501-1515, 2003.

[12] Y. Sawaguchi, E. Furutani, G. Shirakami, M. Araki, and K. Fukuda, "A model-predictive hypnosis control system under total intravenous anesthesia," IEEE Trans. Biomed. Eng., vol. 55, pp. 874-887, Mar. 2008.

[13] R. R. Jaklitsch and D. R. Westenskow, "A model-based self-adjusting two-phase controller for vecuronium-induced muscle relaxation during anesthesia," IEEE Trans. Biomed. Eng., vol. BME-34, pp. 583-594, Aug. 1987. [14] K. T. Olkkola, H. Schwilden, and C. Apffelstaedt, "Model-based adaptive closed-loop feedback control of atracurium-induced neuromuscular blockade," Acta Anaesthesiol. Scnad., vol. 35, pp. 420-423, July 1991.

[15] H. Schwilden and K. T. Olkkola, "Use of a pharmacokinetic-dynamic model for the automatic feedback control of atracurium," Eur. J. Clin. Pharmacol., vol. 40, pp. 293-296, Mar. 1991.

[16] M. Kansanaho and K. T. Olkkola, "Performance assessment of an adaptive model-based feedback controller: Comparison between atracurium, mivacurium, rocuronium and vecuronium," J. Clin. Monitoring Comput., vol. 13, pp. 217-224, Nov. 1996.

[17] J. M. Lemos, H. Magalhães, T. Mendonça, and R. Dionísio, "Control of neuromuscular blockade in the presence of sensor faults," IEEE Trans. Biomed. Eng., vol. 52, pp. 1902-1911, Nov. 2005.

[18] T. Mendonça, J. M. Lemos, H. Magalhães, P. Rocha, and S. Esteves, “Drug delivery for neuromuscular blockade with supervised multimodel adaptive control," IEEE Trans. Control Syst. Technol., vol. 17, pp. 1237-1244, Nov. 2009.

[19] M. Struys, T. De Smet, L. Versichelen, S. van de Vilde, R. van der Brocke, and E. Martier, "Comparison of closed-loop controlled administration of propofol using BIS as a controlled variable versus standard practice controlled administration," Anesthesiology, vol. 95, no. 1, pp. 6-17, 2001.

[20] Q. Hui, W. M. Haddad, V. Chellaboina, and T. Hayakawa, "Adaptive control of mammilary drug delivery systems with actuator amplitude constraints and system time delay," Eur. J. Control, vol. 11, no. 6, pp. 1-15, 2005. [21] C. S. Nunes, T. Mendonça, J. M. Lemos, and P. Amorim, "Feedforward adaptive control of the bispectral index of the EEG using the intravenous anaesthetic drug propofol," Int. J. Adapt. Control Signal Process., vol. 23, pp. 485-503, May 2009. [22] W. M. Haddad, V. Chellaboina, and Q. Hui, Nonnegative and Compartmental Dynamical Systems. Princeton, NJ: Princeton Univ. Press, 2010, pp. 359-522. [23] K. J. Åström and B. Wittenmark, Adaptive Control. Reading, MA: Addison-Wesley, 1989.

[24] C. M. Ionescu, R. De Keyser, B. C. Torrico, T. De Smet, M. M. Struys, and J. E. Normey-Rico, "Robust predictive control strategy applied for propofol dosing using BIS as a controlled variable during anesthesia," IEEE Trans. Biomed. Eng., vol. 55, pp. 2161-2170, Sept. 2008.

[25] G. A. Dumont, A. Martinez, and J. M. Ansermino, "Robust control of depth of anesthesia," Int. J. Adapt. Control Signal Process., vol. 23, pp. 435-454, May 2009. [26] D. V. Caiado, J. M. Lemos, B. A. Costa, L. Paz, and T. F. Mendonça, "A polynomial design approach to robust control of neuromuscular blockade of patients subject to general anesthesia," in Proc. 52nd IEEE Conf. Decision Control, Firenze, Italy, Dec. 10-13, 2013, pp. 133-138.

[27] S. Skogestad and I. Postlethwaite, Multivariable Feedback Control Analysis and Design. Hoboken, NJ: Wiley, 2005.

[28] D. E. Longnecker, D. L. Brown, M. F. Newman, and W. M. Zapol, Anesthesiology. New York: McGraw-Hill Medical, 2008.

[29] J. Keener and J. Sneyd, Mathematical Physiology (Interdisciplinary Applied Mathematics, vol. 8). New York: Springer, 2004, ch. 7, pp. 217-246.

[30] C. Fall, E. S. Marland, J. M. Wagner, and J. J. Tyson, Computational Cell Biology. New York: Springer, 2002.

[31] J. M. Bailey and W. M. Haddad, "Drug dosing control in clinical pharmacology," IEEE Control Syst., vol. 25, no. 2, pp. 35-51, 2005.

[32] K. Glover, "All optimal Hankel-norm approximations of linear multivariable systems and their $\mathrm{L}_{\infty}$-error bounds," Int. J. Control, vol. 39, no. 6, pp. 1145-1193, 1984. 